\title{
Anime, otaku y cintas de vídeo: el formato OVA como catalizador de la complejidad narrativa en la animación japonesa contemporánea
}

\section{Resumen}

En este artículo exploramos la condición de las OVA (las ediciones domésticas de anime) como formato bisagra entre la madurez narrativa del anime en los setenta y la narración compleja de los noventa en adelante. La metodología empleada parte del análisis narratológico inspirado por la poética histórica de los modos de narración realizada por David Bordwell. Así, atendiendo a las categorías de la cognoscibilidad, de la autoconsciencia y de la comunicabilidad, constatamos en los resultados y en las conclusiones que la presunta simplicidad narrativa atribuida al anime no es tal, y que las OVA analizadas -Twilight Q: Meikyū Bukken Fairu Go San Hachi (Mamoru Oshii, 1987), Gunbuster (H. Anno, 1988-89) y Otaku no Video (T. Mori, 1991)— se alzan como hitos clave para el asentamiento de otro tipo de narración en la industria de la animación japonesa contemporánea, uno que lejos de ser formulaico, tiene correspondencia con la estimulante experimentación narrativa internacional de las Ilamadas puzzle films.

\footnotetext{
El autor quiere hacer constar el apoyo de las fuentes de financiación de esta investigación: Conselleria d'Educació, Investigació, Cultura i Esport de la Generalitat Valenciana y Fondo Social Europeo de la Unión Europea (ref. APOSTD/2019/067). Universitat Jaume I, proyectos "Análisis de identidades en la era de la posverdad. Generación de contenidos audiovisuales para una Educomunicación crítica" (ref. 181390.01/1) y "El diseño narratológico en videojuegos: una propuesta de estructuras, estilos y elementos de creación narrativa de influencia postclásica" (ref. 181369.01/1). Ministerio de Ciencia e Innovación del Gobierno de España, proyectos "Nuevos desarrollos socioculturales, políticos y económicos de Asia Oriental en el contexto global" (ref. PID2019-107861B-100) y "Participación ciudadana y medios de comunicación públicos. Análisis de experiencias de co-creación audiovisual en España y en Europa" (ref. RTI2018-093649-B-I00).
}

\author{
Antonio Loriguillo-López ${ }^{1}$ \\ Doctor en Ciencias de la \\ Comunicación \\ Universitat Jaume I \\ Castellón, España \\ Correo electrónico: loriguil@uji.es \\ ๑ orcid.org/0000-0003-3326-6844 \\ Google Scholar
}

Recibido: octubre 23 de 2018

Aprobado: junio 13 de 2020

Palabras clave:

anime, animación, narratología, OVA, vídeo doméstico. 


\section{Anime, otaku, and videotapes: the OVA format as catalyst for narrative complexity in contemporary Japanese animation}

\begin{abstract}
The status of OVAs (domestic editions of anime) is explored in this article as a hinge format between the narrative maturity of anime in the 1970s and the complex storytelling from the 1990s onwards. The methodology used is based on a narratological analysis inspired by the historical poetics of cinema carried out by David Bordwell. Thus, taking into account the categories of knowledgeability, self-consciousness and communicativeness, in the results and conclusions it was found that the presumed narrative simplicity attributed to anime is not such, and that the OVAs analyzed -Twilight Q: Meikyū Bukken Fairu Go San Hachi (Mamoru Oshii, 1987), Gunbuster (H. Anno, 1988-89) and Otaku no Video (T. Mori, 1991)— stand as key milestones in the establishment of another type of storytelling in the contemporary Japanese animation industry, one that, far from being formulaic, corresponds to the stimulating international narrative experimentation of the so-called puzzle films.
\end{abstract}

Key words:

anime, animation, narratology, OVA, VCR. 


\section{Introducción}

¿Es el anime, uno de los productos más reconocidos de la cultura popular de la periferia cinematográfica y televisiva, un arte formulaico? Un repaso a la historiografía producida en Occidente puede hacer pensar que sí. Desde su irrupción en las parrillas televisivas de todo el mundo, la animación comercial japonesa está en el punto de mira crítico de la academia por representar un tipo de circuito comercial que trasciende en forma y fondo la de la forma de animación dominante de la que desciende, el Saturday morning cartoon estadounidense. En consecuencia, el anime es tachado habitualmente de ser "un mero producto a la altura de la comida basura"" (Gan, 2009, p. 39), un subproducto en lo referente al prestigio narrativo que atrae primordialmente a los enfoques culturalistas por su riqueza temática y por las problemáticas representacionales que concurren año a año en su prolífica producción —hasta 356 series producidas solo en el año 2016 — (Masuda et al., 2018).

En este artículo abundamos en la discusión del persistente prejuicio con respecto a la simpleza narrativa del anime atendiendo a tres casos de estudio inéditos, pertenecientes al instante que identificamos como la fase de consolidación de la narración compleja en el anime: la irrupción de las primeras ediciones domésticas y la comercialización bajo el formato OVA (apócope de original video animation). Esta nomenclatura designa aquellos títulos de la animación comercial japonesa producidos directamente para el mercado del vídeo doméstico. El formato vive su época dorada desde mediados de los 80 hasta los primeros años del nuevo milenio, cuando buena parte de los textos más experimentales en términos narrativos encuentran acomodo en el mercado televisivo del narrowcasting ${ }^{2}$, por la irrupción de ventanas en forma de nuevos canales por satélite y por cable. Pese a que recientes investigaciones estudian

\footnotetext{
"Traducción del autor. Original: "A mere fast food-like commercial product".

${ }_{2}^{2}$ Producción de ficción televisiva no para su retransmisión generalista, sino para la multitud de canales especializados que surge tras la extensión de la televisión por cable y las retransmisiones vía satélite.
} 
la rotunda estructura de los comités de producción del media mix del anime (Steinberg, 2012; Hernández Pérez, 2017) como un modelo productivo a seguir por actores comerciales en el resto del mundo por su capacidad productiva crossmedia (Jenkins, 2008) y por su continuada supervivencia en el competitivo entorno del entretenimiento audiovisual contemporáneo (Zahlten, 2014), la época de mayor exposición del formato OVA no goza de un interés investigador consolidado pese a disponer, desde el último lustro, de bases históricas suficientemente sólidas como para establecer nexos de unión con el decisivo cambio que la identidad del anime experimenta durante ese lapso de tiempo concreto. Nuestra intención es la de reclamar la validez de las herramientas propias de la narratología fílmica como instrumentos de interés para la evaluación del anime, una metodología poco favorecida en el ámbito de los Anime Studies internacional, cuyas principales corrientes metodológicas desarrolladas primordialmente desde los estudios literarios, los estudios de Asia Oriental y de los estudios fílmicos (Koulikov, 2015) se aplican a una reducida muestra de títulos de circulación internacional —obras del Studio Ghibli o bien adaptaciones de los shōnen manga de primeros espadas del mundo editorial como Shūeisha o Kōdansha- (Kinsella, 2000), dejando sin cubrir buena parte de la prolífica producción local. Nuestro convencimiento es que un estudio de la narración compleja del anime, su rasgo más eminente, requiere de un análisis narratológico que ya se está llevando a cabo en el ámbito de los estudios cinematográficos y televisivos internacionales.

\section{Marco teórico}

En el patrón historiográfico habitual del anime se señala a tres nombres como los catalizadores de los tres momentos reseñables de la animación japonesa comercial (Nobuyuki Tsugata en Denison, 2016, p. 91): Astroboy (1963-1966), Space Battleship Yamato (1974-1975) y Neon Genesis Evangelion (1995-1996). Astroboy aparece regularmente en las sucesivas historias del 
anime como el origen de la animación comercial japonesa, pero no lo es de su complejidad narrativa. A pesar de su reconocible madurez temática, su narración es eminentemente elemental, en la línea de la formulaicos títulos de Hanna-Barbera. La influyente obra de Tezuka ${ }^{3}$ se adscribe a los rasgos de la convencionalidad catódica: narración episódica, fórmula del monstruo de la semana (Backstein, 2004, p. 117) y argumentos autoconclusivos. Nuestra intención es explorar el espacio liminal entre la época de Yamato y la de Evangelion, dos hitos más estudiados que el prolífico lapso intermedio ocupado por el apogeo del formato OVA. La recuperación de esta época no sólo se está llevando a cabo en el caso de la animación nipona. En uno de los trabajos más provocadores de la historiografía fílmica japonesa reciente, Alexander Zahlten apunta al fin del cine japonés entendido como arte (2017). Su punto de partida no está en las historias canónicas del cine publicadas en Occidente tras la década de los setenta (Tessier, 1999; Richie, 2005; Galbraith, 2009), sino que él aboga por el concepto de géneros industriales:

los géneros definidos como sistemas de constelaciones específicas de textos fílmicos, circulación de capital, prácticas de producción y diseminación (distribución y exhibición) que reproducen similaridad de manera consistente ${ }^{4}$. (Zahlten, 2007, p. 4)

De ellos son representativos las películas de Kadokawa (McDonald, 1989), las pink eiga y el $V$-Cinema ${ }^{5}$, prolíficos definidores de la deriva fílmica de Japón de los últimos 50 años a pesar de su invisibilidad para crítica, academia y públicos fuera de sus fronteras (Elena, 1993). Destacamos este último género (contracción de Video Cinema) por sus correspondencias con el formato que

\footnotetext{
${ }^{3}$ La mención a Osamu Tezuka nos sirve para explicitar las diferencias de madurez del anime con respecto a su forma de expresión más cercana, el manga. Figura clave en la consolidación de las dos formas de arte como verdaderas industrias en Japón, la complejidad narrativa en la que incurre el influyente mangaka en obras previas a las OVA como la saga Fénix (Hi no Tori, 1967-1988) ejemplifica los distintos ritmos y grados de libertad para la experimentación creativa en los dos medios. Títulos como Fénix, pero también las historietas de vanguardia publicadas en revistas manga alternativas como Garo (1964-2002) son ingredientes del caldo de cultivo que conduce a la complejidad narrativa en forma animada.

${ }^{4}$ Traducción del autor. Original: "genres defined as systems of specific constellations of filmic texts, capital circulation, production and dissemination (distribution and exhibition) practices which consistently reproduce similarity".

${ }^{5}$ Activo desde la década de los ochenta y de vital importancia para el desarrollo de la ecología de nuevos medios (reproductores y grabadores de vídeo, ordenadores personales y televisión por cable) en Japón.
} 
consideramos como el catalizador de una "época de confusión" (konmei no jidai, Misono Makoto en Clements, 2013, p. 160) en el anime de la que emerge (y se extiende) la complejidad narrativa que integra sus títulos posteriores más reconocidos. Como el V-Cinema, las OVA se distribuyen en su origen a través de ediciones de videocasetes de vídeo. La irrupción de este formato ${ }^{6}$ revoluciona la narración del anime en una época en el que estudios de reciente creación —y entre cuyos equipos artísticos comienzan a aparecer la primera generación de animadores que crecen con anime maduros como Yamato o Gundam en televisión ${ }^{7}$ - experimentan como nunca para dar con la fórmula de hacer rentables sus propuestas alternativas al mainstream. El número de estudios con la audacia de producir un tipo de anime distinto, sin la presión de un retorno económico mínimo impuesta por grandes inversores en comités de producción, ni los filtros de exhibidores ni de cadenas televisivas, redunda en una libertad creativa que conlleva una mayor variedad y oferta para el mercado del anime doméstico, mucho menos voluminoso y con mayor flexibilidad para asumir riesgos.

Este contexto ayuda a entender la emergencia de unas normas narrativas internas. En general, destacamos una mayor tolerancia para la explicitación de la violencia y la "democratización del espectáculo del sexo" (Elena, 2000, p. 14) (vía explotation del fan service) pero, también, advertimos cierto espacio para la experimentación con modos de narrar más ambiguos que configuran la identidad y la experiencia OVA, a nuestro parecer sesgada en la actualidad por la metonimia que desde crítica y públicos occidentales se realiza hacia

\footnotetext{
${ }^{6}$ Los formatos VHS y Betamax se presentan en 1975 y 1976, respectivamente.

${ }^{7}$ Los verdaderos orígenes de la madurez temática y narrativa en el anime. Situada entre 1974 y 1983, esta época define a una primera generación de otaku, niños y adolescentes entusiastas que crecen viendo en la televisión series como Yamato y Gundam. Si Yamato es con frecuencia designada como la introductora de la complejidad argumental propia de la ciencia-ficción dura a públicos juveniles, Gundam representa el éxito de la complejidad tanto argumental como formal por tratarse del primer serial complejo del anime. La prolífica saga mecha es un ejemplo claro de la explotación comercial del rasgo complejo a través de las alianzas producidas como resultado de la constitución de comités de producción del media mix: sus numerosas temporadas encuentran automática correspondencia en los sucesivos robots a escala articulados comercializados por Bandai en cada nueva iteración animada.
} 
el binomio anime/hentai ${ }^{8}$. La libertad de maniobra del género pink eiga tal y como es descrito por Zahlten es similar a la de este nicho del anime que explota también gracias a la difusión de las ediciones domésticas. La circulación internacional de exitosas OVA como Cream Lemon (1984-2006) y las adaptaciones de los manga eróticos de Toshio Maeda como las series Urotsukidōji (1987-1994) o La Blue Girl (1992-1993) son un factor decisivo para entender tanto el celo hacia la animación japonesa comercial por parte de la opinión pública (Williams, 2006), como la atracción académica de estudio desde enfoques culturalistas hasta nuestros días (McCrea, 2008; Piñón Perales, 2010; Pett, 2016). Más allá del hentai, la irrupción de la cinta de video transforma radicalmente la manera de consumir anime, pero también introduce cambios esenciales en su producción que permiten la inserción de la complejidad narrativa. El boom del "anime adulto" (en Denison, 2016, p. 92), como lo bautiza el historiador del medio Tsugata, proviene inequívocamente del vídeo, la llave para la atomización de los distintos nichos del anime frente al mainstream, constreñido por las cadenas de televisión generalistas a lo que él denomina "anime familiar". Señalado por Denison (2016) como "los límites más oscuros del anime y de los cuerpos más explícitos y extremos"9 (p. 59), el formato OVA es, para teóricos como Tsugata, el embrión de lo que entendemos como anime complejo.

La aparición de la OVA no solo altera la identidad del anime en el mercado local. Diversos autores emparentan la expansión transnacional del anime con el desarrollo tecnológico de la distribución nacional e internacional de anime — primero bajo la forma de VHS, luego de ediciones en DVD y, finalmente con la distribución por internet- y los consecuentes cambios estructurales

\footnotetext{
${ }^{8}$ Aunque, como sugiere Napier, el caso de la filmografía de directores adscritos al género como Yoshiaki Kawajiri, "el género podría ser descrito más apropiadamente como animación 'de ciencia-ficción y de lo oculto con imágenes y temas explícitamente sexualizados'”. (Napier, 2001 p. 341) Traducción del autor, original: "the genre might more appropriately de described as 'science fiction and occult animation with explicitly sexualized imagery and themes'".

9 Traducción del autor. Original: "anime's darker fringes and more explicit and extreme bodies"
} 
en su industria (Cubbison, 2005). La progresiva adquisición de reproductores/ grabadores de vídeo doméstico por parte de los seguidores de anime eleva su listón de exigencia en sus demandas, incitando a la recuperación de anime descatalogado y a ciertos cambios en las responsabilidades creativas de los estudios. Muchas de las reorganizaciones de personal en estos años derivan de un creciente reconocimiento a los artistas detrás de las producciones animadas, nombres auspiciados al estatus de "autores" desde las tribunas que conforman de las revistas especializadas en animación japonesa comercial (como Animage o la influyente Newtype) que se consolidan también en esta época. De entre estos nombres reseñamos, por sus aportaciones a la complejidad narrativa en la animación comercial japonesa, al director Mamoru Oshii y al colectivo Studio Gainax.

\section{Método}

A lo largo del texto entendemos como complejidad narrativa aquella narración cuya coherencia comunicativa es deliberadamente ofuscada. Para la evaluación de la manera en la que la narración fílmica transmite información partimos en nuestra metodología de las proyecciones sobre las maneras de narrar y su cruce con una poética histórica del cine planteados por David Bordwell (1996). A diferencia de otras aportaciones de análisis fílmico integral (Aristizábal Santa y Pinilla-Rodríguez, 2017), Bordwell emplea tres categorías a la hora de caracterizar las estrategias de transmisión de información al espectador por parte de la narración con el fin de acometer con propiedad el estudio de

la forma en que el estilo del filme y la construcción del argumento manipulan el tiempo, el espacio y la lógica narrativa para permitir al espectador construir un desarrollo específico de la historia. (Ibidem, p. 61)

La primera de ellas es la cognoscibilidad, dependiente, a su vez del grado de restricción — ¿ ise limita el conocimiento de la narración a los saberes 
de un personaje concreto? La restricción presenta entonces un grado alto. Por el contrario, estamos ante un bajo grado de restricción en el caso de filmes con protagonismo coral- y del grado de profundidad de ese conocimiento - que guarda relación directa con la subjetividad de saberes: a mayor profundidad, mayor subjetividad (unaalta profundidad permitea los espectadores ver procesos mentales subjetivos como ensoñaciones y delirios)—. La segunda categoría es la autoconsciencia, la mostración de un reconocimiento por parte de la narración de estar interpelando a un público. Las marcas enunciativas son señales evidentes de autoconsciencia. La gradación de la autoconsciencia de este tipo de marcas (entre las que se incluyen los movimientos de cámara, las repeticiones, las rupturas de la cuarta pared o las voces over) depende en buena medida de cómo la revelación de la existencia de la narración a los espectadores condiciona al argumento dentro de las convenciones genéricas y de los modos de producción (p.ej. pese a su autoconciencia, los primeros planos de las estrellas son una convención del cine de los estudios del Hollywood clásico). Por último, Bordwell se refiere a la comunicabilidad como el grado de efectiva comunicación de los saberes al espectador que la narración se permite en función de una estimación de su máximo posible. Este máximo queda establecido en buena medida por las graduaciones de las dos categorías anteriores. Esta última es una categoría especialmente útil para ubicar esas lagunas de la narración, consecuencia de una desviación de la norma interna de comunicabilidad de un filme, y para sopesar las motivaciones transtextuales, como el género (suspense en el policíaco, sorpresa en el terror), respecto a las demandas estructurales intrínsecas. Aunque, como el propio Bordwell advierte, "[e]n general, las películas narrativas modulan constantemente la amplitud y profundidad del conocimiento de la narración" (Ibidem, p. 58), poner atención al desarrollo de estas modulaciones, en especial en las fases más complejas del film, ayuda a arrojar luz sobre la ambigüedad de la etiqueta de "complejidad narrativa". 
Mediante la comparativa de las variables narrativas de nuestros objetos de estudio pretendemos atender a la autonomía de la narración compleja con respecto a las convenciones del cine clásico detalladas en la tabla 1.

Tabla 1. Resumen de las categorías de estrategias narrativas propuestas por Bordwell (1996) para el modo de narración clásico

\begin{tabular}{ccccc}
\hline & \multicolumn{2}{c}{ Cognocibilidad } & Autoconsciencia & Comunicabilidad \\
\cline { 2 - 3 } & Restrictividad & Profundidad & & \\
\hline Clásico & Baja & Alta & Baja-moderada & Moderada \\
\hline
\end{tabular}

Fuente: elaboración propia.

Frente al modo de narración clásico, una de las más boyantes tendencias en el campo de la experimentación narrativa en el seno del postclasicismo audiovisual comercial es lo que diversos autores etiquetan como puzzle films (Buckland, 2009a; Panek, 2006; Kiss \& Willemsen, 2017): "un ciclo popular de filmes que desde los noventa rechaza las técnicas de la narración clásica y las sustituye por narración compleja"10 (2009b, p. 1). Esta etiqueta viene a representar a aquellos filmes -como Corre Lola Corre (T. Tykwer, 1998), Memento (C. Nolan, 2000) u Oldboy (P. Chan-wook, 2003)— cuya enmarañada syuzhet provoca una comunicabilidad baja que redunda en una disonancia cognitiva similar a la de un rompecabezas — p.ej. elementos ordenados de forma no lineal, mise en abyme no jerarquizadas entre sí, causalidad diluida en grandes lagunas de conocimiento sobre la diégesis, etc.- que los espectadores deben esforzarse en ajustar en un relato coherente.

Nuestra hipótesis es que las OVA que proponemos como títulos paradigmáticos de la narración compleja en el anime _-Twilight Q: Meikyū Bukken Fairu Go San Hachi (Twilight Q 2: Mystery Article File 538, M. Oshii, 1987), Gunbuster

\footnotetext{
${ }^{10}$ Traducción del autor. Original: "a popular cycle of films from the 1990 s that rejects classical storytelling techniques and replaces them with complex storytelling".
} 
(Toppu o Nerae!, H. Anno, 1988-1989) y Otaku no Video (T. Mori, 1991)_ se adscriben a parámetros alejados de las convenciones narrativas del modo de narración clásico, valores propios de las puzzle films.

\section{Casos de estudio}

\section{Mamoru Oshii y Twilight Q}

La razón por la que he podido continuar mi carrera está en esa habilidad de conversión entre los fans japoneses del anime. Mis obras han sido reproducidas y re-publicitadas cada vez que un formato se convierte a otro medio. En otras palabras, he recibido derechos de autor cada vez, lo que a menudo me sustentó en plena escasez de trabajo... De hecho, todas mis obras se han distribuido en video, LD y DVD y me permitieron comprar una casa como el resto de la gente ${ }^{11}$. (Wada-Marciano, 2012, p. 82)

Mamoru Oshii atribuye su éxito como autor a la remediación comercial de sus obras. Se trata de una afirmación consecuente en el caso de un cineasta cuya carrera se escinde en dos precisamente con la considerada como la primera OVA del anime: Dallos (1983-1984). Inconsecuente artísticamente, el valor de Dallos se reduce a ser el precedente de los títulos de más compleja narración de la década dorada del formato OVA. Oshii disfruta en esta época de una carrera comercial como director de la primera centena de episodios de la adaptación del manga superventas de Rumiko Takahashi, Urusei Yatsura (Lum, la chica invasora) (1981-1986) y de sus dos primeros largometrajes: Urusei Yatsura Movie 1: Only You (1983) y la compleja Lamu Beautiful Dreamer. La película (1984). Antes de trabajar a pleno rendimiento con Headgear ${ }^{12}$, Oshii contribuye a un proyecto denominado Twilight $Q$, una serie de OVA imbuidas por el espíritu de antologías como The Twilight Zone, que sirven como escaparate para la nueva

\footnotetext{
${ }^{11}$ Traducción del autor. Original: "The reason that I have been able to continue my career is in this high 'convert-ability' among the lapanese anime fans. My works have been reproduced and re-promoted each time when a medium's format is converted. In other words, I received rovalties each time, which often supported me when I had a scarcity of work... Indeed, all my works have been distributed in video, LD, and DVD, and they allowed me to buy a house like other people".

${ }^{12}$ Grupo de profesionales desengañados del anime con los que impulsa proyectos personales de manera cooperativa para conservar, entre todos, el control de creaciones como la exitosa saga Patlabor.
} 
camada de artistas del anime a finales de 1980 (Sevakis, 2007). Oshii escribe y dirige la, a la postre, última entrega de la serie tras la cancelación del proyecto: Twilight Q: Meikyū Bukken Fairu Go San. Erigida por una de las muestras de experimentación con la narrativa compleja, su condición de precedente la hace imprescindible en un repaso a la narración del medio.

En su secuencia de apertura, un avión de Japan Airlines (correspondiente al vuelo 538) muta en pleno vuelo en una carpa decorativa descomunal. En una radio se anuncia la desaparición del avión, punto de enlace con el cuerpo de la narración, articulado por el relato en primera persona de un modesto detective privado en el Tokio contemporáneo con un extraño encargo: investigar a un hombre y a su presunta hija pequeña en un desvencijado apartamento. Tras infructuosos avances —la búsqueda documental no es concluyente y los investigados no hacen más que comer, dormir y excretar por la ventana-, el detective entra finalmente en la estancia para encontrar a la niña durmiendo sobre su futón con una carpa viva a su lado. En la mesa encuentra unas páginas dirigidas a él, "su sucesor": una historia de otro detective privado que sigue, paso por paso, el mismo patrón que la suya. El relato concluye con revelación. El detective se quita las gafas de sol y mira a cámara desafiante: se ha convertido en el hombre que acompaña a la niña.

La irrupción del componente fantástico tensa las normas internas del noir al que la narración parece adscribirse, una tensión aún incrementada por la revelada autoconsciencia del epílogo. En la mañana siguiente, el detective está aparentemente en el papel del hombre adulto a cargo de la niña. En la radio informan de la desaparición del vuelo JAL 538, un hecho que rima con el prólogo... hasta que el hombre detiene en radiocasete donde estaba sonando una cinta con esa información. El hombre habla animadamente por teléfono con su editor, al que le está vendiendo la idea para una historia titulada "El caso eterno": un detective descubre que tanto él mismo como el hombre al 
que sigue no son más que una creación divina para cuidar de una niña-Diosa. Tras enojarse por la reacción del editor (que le pide una historia más normal), el hombre se dispone a arreglar un pescado para comer. Tras hacer una hendidura en su vientre, un avión que sobrevuela los cielos se abre por la mitad.

A pesar de la animación limitada, el uso inteligente de los recursos como la voz over (adscrita al género detectivesco) y el uso de flashbacks con apenas acción apoyan este aparente juego de muñecas rusas, basado en recursos familiares para los puzzle films como la severa restricción en el protagonista focalizador, la alta profundidad en sus saberes y la autoconsciente disposición de lagunas de información - ¿qué une al avión inicial con la historia del detective?, ¿es una metáfora del cuidado de una diosa en ciernes por parte de un ser humano?- , es rematado por una baja comunicabilidad que hace de esta OVA uno de los títulos más ambiguos de la obra de Oshii.

\section{Studio Gainax}

Gainax supone el más notable salto de fans a productores desde los clubs universitarios de apreciación de la ciencia-ficción (Loriguillo-López, 2019) hasta la constitución del estudio que más tarde alumbraría uno de los anime complejos por antonomasia, Evangelion. La notoriedad alcanzada por sus cortometrajes animados producidos para dos ediciones de la convención anual japonesa de ciencia-ficción (Nihon SF Taikai) celebradas en Osaka en los años 1981 y 1983 y la red de contactos tejida entre distintos actores de la industria propulsa las carreras de los diseñadores de personajes Yoshiyuki Sadamoto y Takami Akai, del guionista (y líder de opinión otaku) Toshio Okada, y de los directores Hiroyuki Yamaga, Shinji Higuchi y Hideaki Anno. Desde entonces, estos individuos han quedado establecidos como iconos de la borrosa línea entre el fan y el profesional que caracteriza a las convergencias creativas entre industria y audiencias del anime (Condry, 2013). 
Además de desdibujar la frontera entre el fan y el profesional, Gainax tiene para el imaginario colectivo una asociación indisoluble con la complejidad narrativa. Gainax destaca por la experimentación narrativa de sus anime originales —no realizan ninguna adaptación ajena a sus propios materiales hasta su participación en Kare Kano (TV Tokyo: 1998-1999)_, fruto de la autonomía creativa que otorgan tanto la independencia de las propiedades de otras franquicias como el sustento generado desde su propio emprendedurismo en el merchandising, los videojuegos adultos y los garage kits ${ }^{13}$ a través de la compañía hermana, General Products.

\section{Otaku no Video}

Gainax muestra con orgullo sus orígenes en su propia autobiografía dramatizada, Otaku no Video (T. Mori, 1991), una OVA en dos partes centrada tanto en el encuentro de estos autodenominados otakings ${ }^{14}$ como en su meteórico ascenso a la producción profesional en el trascurso de 1982 a 1991, incluyendo su aprendizaje a marchas forzadas para la animación con acetatos. La autoconsciencia es explícitamente alta si, además, se tiene en cuenta que, entre el metraje animado, se incluyen falsas estadísticas ${ }^{15}$ y entrevistas en imagen real bajo la forma del falso documental a otaku adultos que satirizan el estereotipo de una de las tribus urbanas más vilipendiadas en el Japón de los primeros años de 1990. El intertítulo "Retrato de un otaku" sobre las barras de un sistema de vídeo aparece como cabecera de las secuencias de entrevistas de imagen real, rasgo también de evidente de autoconsciencia.

\footnotetext{
${ }^{13}$ Figuras de modelado de tiradas muy limitadas producidas por fans para fans (muchas veces en talleres ubicados en garajes). Su alto coste es proporcional a la complejidad de su montaje que demanda destreza con la manipulación de resinas, pinturas y barnices.

${ }^{14}$ Entusiastas de La guerra de las galaxias, el tokutatsu y del anime Yamato.

${ }^{15}$ Que, a su vez, revelan un gran sentido del humor por parte de sus autores, totalmente conscientes de representar al colectivo (p.ej. "el $100 \%$ de los otaku son vírgenes").
} 


\section{Gunbuster}

La experimentación narrativa de Gainax tiene de nuevo su primer banco de pruebas en el formato OVA: Gunbuster. Su clímax está centrado en los estragos dramáticos que la dilatación temporal de los viajes espaciales ${ }^{16}$ tiene sobre las relaciones entre los personajes principales, jóvenes adolescentes separadas de la Tierra para combatir en una campaña contra la amenaza extraterrestre y cuya vuelta no se produce hasta 12.000 años después de tiempo terráqueo. De esta manera, y en la línea de lo que ofrecen en su primer largometraje -Royal Space Force: The Wings of Honneamise (H. Yamaga, 1987)—, Gainax parte temáticamente de la herencia de madurez del medio de etapas anteriores. Sucesora de Yamato y de Gundam, Gunbuster representa la progresiva consolidación de la ciencia-ficción dura en el anime que, lejos de versiones celebratorias de batallas para el lucimiento de los diseños mecánicos, incide en presentar un conflicto armado del que nunca se pueden conseguir victorias absolutas. La narración en Gunbuster es altamente autoconsciente de su condición de obra por fans y para fans pero, más allá de la temática, de las condiciones de producción y del fan service, añade además interesantes momentos de complejidad.

En el teaser del episodio \#01x04: Launch!! The Incomplete Ultimate Weapon!, una versión chibi de la protagonista, Noriko Takaya, explica que el guion de los siguientes episodios planificados no está acabado: “¡El proyecto del siguiente episodio se ha aprobado tan rápido que aún no tenemos el guion listo! [...] Sólo tenemos confirmados los títulos de los próximos dos episodios". Esta suerte de aviso para navegantes parece avanzarse a la especulación que sigue tras la salida al mercado del último episodio de la OVA, \#01x06: At the End of Eternity. Su principal particularidad es que el metraje está en blanco y negro. Pero al contraste inicial con respecto al metraje, a todo color,

${ }^{16}$ Un tropo habitual en el cine de ciencia-ficción comercial estadounidense desde El planeta de los simios (Planet of the Apes, F. J. Schaffner 1968) hasta Interstellar (C. Nolan, 2014). 
del resto de la serie le sucede la sorpresa cuando la esperada batalla final, lejos de cumplir con el estándar de los apogeos animados de los mecha ${ }^{17}$, es brevemente recreada mediante una secuencia de montaje fugaz de animación limitada cercana a la kinestasis (Laybourne, 1979) en la que se recurre a fundidos, panorámicas, filtros y sombreados para animar ilustraciones (que también contribuyen a la sensación de movimiento al dar uso a los códigos cinéticos del manga) de la destrucción de las flotas espaciales humanas y de las formas orgánicas alienígenas.

En Gunbuster ubicamos el inicio de una rumorología de la sospecha sobre la relación entre las intenciones creativas de Gainax y su presunta incompetencia en lo relativo a las cuestiones presupuestarias: ¿responde la narración del episodio, radicalmente transgresora con los postulados del género mecha por privilegiar la psicología de sus personajes ante la tragedia al espectáculo de la batalla, a una intencionada voluntad discursiva, como defiende el locuaz Okada (Viz Communications, 1996), aquí guionista? ¿Se debe al agotamiento de recursos para cerrar el proyecto (y a las documentadas dificultades de Anno para concluir sus series $\left.{ }^{18}\right)$ ? ¿U obedece la experiencia estética resultante al gusto de Anno por la "imagen-tiempo" deleuziana en lo que Thomas Lamarre acuña como "hyperlimited animation" (Lamarre, 2009, p. 200)? Toda esta serie de factores sientan en el imaginario colectivo de las comunidades otaku un precedente artístico-industrial que es jocosamente referido por crítica y espectadores como definidor del tropo "Gainax Ending" (TV Tropes, s.f.), una etiqueta asimilable a las de puzzle films o complex TV y que, como en sus casos, se refiere a la tendencia a la ruptura de la unidad mimética de la syuzhet. Pese a la relevancia de Gunbuster, el término y la fama del estudio comienza su circulación internacional (McKevitt, 2010) con Evangelion.

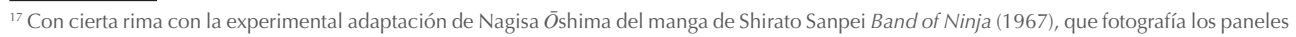
ilustrados y a los que les añade una banda sonora.

${ }^{18}$ Una década más tarde, Anno abandona a mitad la producción de Kare Kano diagnosticado de depresión.
} 


\section{Conclusiones}

A través de este repaso constatamos que, desde los postulados de la narratología, los casos de anime analizados cuentan con una narración compleja que se mueve en los parámetros de la no linealidad de las puzzle films con unos años de antelación a la consolidación de la tendencia en la década de los noventa. Como vemos en la tabla 2, los títulos analizados divergen de los parámetros del modo de narración clásico de manera en que la media de comunicabilidad es consistentemente menor a la alta claridad de los convencionalismos narrativos comerciales. El parámetro de mayor diferencia es la autoconsciencia, un rasgo claramente marcado en el anime como exponente del modo de narración postclásico.

Tabla 2. Resumen de las categorías de estrategias narrativas propuestas por Bordwell (1996) para el modo de narración clásico y para las OVA analizadas

\begin{tabular}{ccccc}
\hline & \multicolumn{2}{c}{ Cognocibilidad } & Autoconsciencia & Comunicabilidad \\
\cline { 2 - 3 } & Restrictividad & Profundidad & & \\
\hline Clásico & Baja & Alta & Baja-moderada & Moderada \\
\hline Twilight $Q$ & Alta & Alta & Alta & Baja \\
\hline Otaku no Video & Baja & Alta & Explicitamente alta & Moderada \\
\hline Gunbuster & Alta & Moderada & Alta & Moderada \\
\hline
\end{tabular}

Fuente: elaboración propia.

Nuestra aportación es evidentemente limitada tanto en la muestra de objetos de estudio como en su alcance. El enfoque que proponemos es potencialmente extensible a una poética histórica del anime complejo, una empresa que requiere del cuidadoso inventariado de las decisiones creativas y narrativas en la era de la narración postclásica y su razón de ser en el seno de una industria mediante puntualizaciones desde la economía política de la comunicación. 
Por ejemplo, la narración más explícitamente autoconsciente se integra en el anime contemporáneo en títulos de gran popularidad tan dispares como Paranoia Agent, Gintama o Shirobako. La primera, creada por el célebre auteur Satoshi Kon, se adscribe al modelo de narración de la televisión compleja con rasgos como la "metanimación" de su décimo episodio (\#01x10: Maromi Madoromi), en el que unos misteriosos asesinatos alteran el flujo de trabajo de un anime televisivo. En la segunda, una de las más longevas comedias originadas en la revista Weekly Shōnen Jump durante los años 2000, incide en la naturaleza del dispositivo animado en su episodio \#02x94: Densha ni noru toki wa kanarazu ryōte o tsurikawani, con la descomposición de una secuencia inicial en una sucesión de fotogramas clave sin intercalar para dar la sensación de que se trata de una simple prueba de animación. Finalmente, en la tercera, los espectadores asistimos a la trastienda de las producciones animadas hechas en Japón mediante los primeros pasos como profesionales en la industria del grupo de jóvenes protagonistas. Estos primeros pasos están marcados por abundantes guiños a figuras reconocidas de la industria, caso de un trasunto del propio Hideaki Anno (Mitsuaki Kanno) en el episodio \#01×12: Exodus Christmas. Esta aproximación al anime complejo no es un proyecto autárquico con respecto a la producción cultural del resto del mundo, ya que funciona como un caso de estudio paradigmático de una tendencia global en la que identificamos una aparente contradicción: la aparición de la complejidad narrativa en el seno de la producción audiovisual contemporánea. ¿Debe interpretarse esta improbable alianza como un nuevo episodio de la reacción mediafóbica de los productores de cine y televisión mainstream ante la interactividad de los videojuegos? ¿Se trata de una simple concesión al gusto por la experimentación de unos nichos de mercado concretamente rentables en el contexto del mercado fragmentado de la industria del entretenimiento japonesa? ¿O estamos ante la hibridación definitiva entre los modos narrativos del cine de arte y ensayo y los de la comercialidad, otrora enfrentados? El amplio espectro de la animación comercial japonesa, sin la losa de aspirar 
a la etiqueta "para toda la familia" que pesa sobre la animación comercial, comprende una estimulante diversidad de géneros y tendencias y, entre ellas, una serie de títulos en los que la narración compleja, no formulaica, tiene un papel central.

\section{Referencias}

Aristizábal-Santa, J. y Pinilla Rodríguez, Ó. (2017). Una propuesta de análisis cinematográfico integral. Revista Kepes, 16, 11-32.

Backstein, K. (2004). Flexing Those Anthropological Muscles: X-Files, Cult TV, and the Representation of Race and Ethnicity. En S. Gwenllian-Jones y R. E. Pearson (Eds.), Cult Television (pp. 115-145). Minneapolis, USA: University of Minnesota Press.

Bordwell, D. (1996). La narración en el cine de ficción. Barcelona, España: Paidós.

Buckland, W. (Ed.) (2009a). Puzzle films: complex storytelling in contemporary cinema. Malden, Estados Unidos: Wiley-Blackwell.

Buckland, W. (2009b). "Introduction: Puzzle Plots". En W. Buckland (Ed.), Puzzle films: complex storytelling in contemporary cinema (pp. 1-12). Malden, USA: Wiley-Blackwell.

Clements, J. (2013). Anime: A History. London, UK: BFI \& Palgrave Macmillan.

Condry, I. (2013). The Soul of anime: collaborative creativity and Japan's media success story. Durham, USA: Duke University Press.

Cubbison, L. (2005). Anime Fans, DVDs, and the Authentic Text. The Velvet Light Trap, 56(1), 45-57.

Denison, R. (2016). Anime: A Critical Introduction. Londres, UK: Bloomsbury. 
Elena, A. (1993). En el país de Godzilla: una introducción al cine japonés. Nosferatu. Revista de cine, 11, 4-13.

Elena, A, (2000). La puerta del infierno: tres notas sobre la circulación del cine pornográfico. Secuencias, 12, 7-22.

Galbraith, S. (2009). Cine japonés. Köln, Alemania: Taschen.

Gan, S. H. (2009). To Be or Not to Be. The Controversy in Japan over the 'Anime' Label. Animation Studies, 4, 35-43.

Hernández-Pérez, M. (2017). Manga, anime y videojuegos. Narrativa crossmedia japonesa. Zaragoza, España: Prensas Universitarias de Zaragoza.

Jenkins, H. (2008). Convergence culture: la cultura de la convergencia de los medios de comunicación. Barcelona, España: Paidós.

Kinsella, S. (2000). Adult manga: culture and power in contemporary Japanese society. Richmond, USA: Curzon.

Kiss, M. \& Willemsen, S. (2017). Impossible Puzzle Films. A Cognitive Approach to Contemporary Complex Cinema. Edinburgh, UK: Edinburgh University Press.

Koulikov, M. (2015). Researching the Business of Anime. Resources and Thoughts. Recuperado de https://animemangastudies.wordpress.com/2015/01/02/ researching-the-business-of-anime-resources-and-thoughts/

Laybourne, K. (1979). The animation book: a complete guide to animated filmmaking, from flip-books to sound cartoons. Nueva York, USA: Crown Publishers.

Loriguillo-López, A. (2019). Genealogía de las prácticas comunicativas de los "otaku": evolución de la tecnología audiovisual y de la cultura fan en el consumo del "anime". Arte, Individuo y Sociedad, 31(4), 917-930. 
Masuda, H., Sudo, T., Rikukawa, K., Mori, Y., Ito, N. \& Kameyama, Y. (2018). Anime Industry Report 2017 Summary. Recuperado de http://aja. gr.jp/?wpdmdl=1202.

McCrea, C. (2008). Explosive, Expulsive, Extraordinary: The Dimensional Excess of Animated Bodies. Animation, 3(1), 9-24.

McDonald, K. (1989). Popular Film. En R. Gid Powers y H. Kato (Eds.), Handbook of Japanese Popular Culture (pp. 97-126). Westport, USA: Greenwood Press.

Mckevitt, A. C. (2010). 'You Are Not Alone!': Anime and the Globalizing of America. Diplomatic History, 34(5), 893-921.

Napier, S. J. (2001). The Frenzy of Metamorphosis. The Body in Japanese Pornographic Animation. En D. Washburn \& C. Cavanaugh, Carole (Eds.), Word and Image in Japanese Cinema (pp. 342-365), Cambridge, Estados Unidos: Cambridge University Press.

Panek, E. (2006). The Poet and the Detective: Defining the Psychological Puzzle Films. Film Criticism, 31, 62-88.

Pett, E. (2016). 'Blood, guts and Bambi eyes': Urotsukidoji and the Transcultural Reception and Regulation of Anime. Journal of British Cinema and Television, 13(3), 390-408.

Piñón Perales, F. (2010). La representación grotesca en el anime. Perífrasis, 2(1), 95-113.

Richie, D. (2005). A Hundred Years of Japanese Film: A Concise History, with a Selective Guide to DVDs and Videos. Tokio, Japón: Kodansha International.

Sevakis, J. (2007). Twilight Q. Recuperado de http://www.animenewsnetwork. com/buried-treasure/2007-11-08 
Steinberg, M. (2012). Anime's Media Mix: Franchising Toys and Characters in Japan. Minneapolis, USA: University of Minnesota Press.

Tessier, M. (1999). El Cine japonés. Madrid, España: Acento.

Thanouli, E. (2009). Post-classical cinema: an international poetics of film narration. Nueva York, Estados Unidos: Wallflower Press.

TV Tropes. (s.f.). Gainax Ending. Recuperado de http://tvtropes.org/pmwiki/ pmwiki.php/Main/GainaxEnding.

Viz Communications. (1996). Otaking. Toshio Okada at Anime America '96. Recuperado de http://web.archive.org/web/20001217033900/http://www. j-pop.com:80/anime/archive/feature/04_gal_999/otaking.html

Wada-Marciano, M. (2012). Japanese Cinema in the Digital Age. Honolulu, Estados Unidos: Hawai'i University Press.

Williams, K. L. (2006). The Impact of Popular Culture Fandom on Perceptions of Japanese Language and Culture Learning: The Case of Student Anime Fans. University of Texas, UEA.

Zahlten, A. (2007). The role of genre in film from japan: Transformations 1960s-2000s. Johannes Gutenberg-Universitaet Mainz, Alemania.

Zahlten, A. (2014). Media Mix and the Metaphoric Economy of World. En D. Miyao (Ed.), The Oxford Handbook of Japanese Cinema (pp. 438-456). Oxford, Reino Unido: Oxford University Press.

Zahlten, A. (2017). The End of Japanese Cinema: Industrial Genres, National Times, and Media Ecologies, Durham, USA: Duke University Press.

Como citar: Loriguillo-López, A. (2020). Anime, otaku y cintas de vídeo: el formato OVA como catalizador de la complejidad narrativa en la animación japonesa contemporánea. Revista KEPES, 17(22), 345-366. https://doi.org/10.17151/kepes.2020.17.22.13 